\title{
Editorial
}

\section{ATENCIÓN DEL PARTO Y REFORMA DE LA SALUD}

Las autoridades de salud han manifestado su interés en incluir la atención del parto en el régimen de "Garantías Explícitas de Salud (AUGE GES), que la reforma en curso ofrece a la población del país. Bajo ese predicamento, el Ministerio ha hecho circular el documento Guía Clínica Atención Humanizada del Trabajo de Parto y Parto (2007).

En la actualidad se verifican menos de 240.000 nacimientos anuales en el país, la inmensa mayoría de ellos con control prenatal adecuado y atendidos profesionalmente $(99,8 \%)$. Nuestra tasa de partos prematuros es menor que la de muchos de los países más desarrollados (la mitad al menos de la de Estados Unidos). Nuestras cifras de mortalidad materna y perinatal son comparables a las de los países mas desarrollados del mundo, cuyo gasto del PIB en salud es muy superior al de Chile. Es cierto que la mortalidad materna permanece relativamente estacionaria los últimos años y que tenemos metas que alcanzar en ese indicador, pero lo fundamental es que nuestros resultados son muy buenos. Chile ocupa, de hecho, el quinto lugar en salud infantil a nivel mundial, y el lugar 19 en salud materna (Lancet 2006), debiendo destacarse que estos resultados se han alcanzado sin legalización del aborto; con porcentajes de anticoncepción, entre las mujeres en edad fértil, menores que en muchos países y, además, como se mencionó, con un gasto en salud proporcionalmente inferior.

Por otra parte, los resultados mencionados no son a expensas de lo que se hace en los hospitales universitarios o en las grandes clínicas privadas, porque un porcentaje mayoritario de la población se atiende en los hospitales pertenecientes al sistema público.

Las autoridades consideran, con razón, que dos tareas pendientes son la alta tasa de parto por operación cesárea (mayor en el sistema privado), y el apoyo y personalización de los cuidados maternos y neonatales durante el trabajo de parto. La guía clínica en circulación plantea como objetivo principal el "garantizar el acceso de toda la población de gestantes en Chile a una asistencia profesional adecuada en el trabajo de parto y parto. El objetivo final es lograr un parto seguro, personalizado y humano".

A continuación la guía clínica analiza el concepto de humanización, y luego describe algunas conductas sugeridas, señalando para ellas su nivel de evidencia y grado de recomendación disponible en la literatura médica. Destacan el apoyo emocional continuo (reduciría las probabilidades de parto operatorio, cesárea y anestesia); el uso de monitorización electrónica continua de la FCF (no es recomendable); diferentes alternativas farmacológicas de analgesia-anestesia para el trabajo de parto así como métodos alternativos de alivio del dolor durante la dilatación; libertad de posición y movimiento durante todo el parto; uso restrictivo de la episiotomía; eliminación del enema evacuante y rasurado genital; y contacto temprano, piel a piel, de las madres y sus recién nacidos.

Cabe preguntarse, sin embargo, cual es la necesidad de incorporar la atención del parto al GES (el embarazo y parto no son una patología per se), en circunstancias de que los resultados del país en salud materna y perinatal nos colocan, como ya se dijo, entre los países mas avanzados del mundo, y en circunstancias de que hay muchas otras condiciones que no pueden presentar a nivel local los mismos resultados, sino que además, plantean problemas de equidad que, de nuevo, en el área materno perinatal se han reducido muy significativamente en los últimos años. ¿Se pretende entonces introducir "humanización"? ¿Puede una ley modificar conductas que deben ser aprendidas de los maestros?

No debemos olvidar que una de las condiciones para la atención a través del GES, exige el ingreso a la "Red de Prestadores" establecida por 
el seguro de salud, lo cual lógicamente limita al extremo la libre elección por parte de los beneficiarios (no todos los médicos forman parte de la red). Por otra parte, la inclusión de más patologías en el sistema de garantías ha significado un aumento en las cotizaciones de salud de los seguros privados (ISAPRES). Es probable que desde el punto de vista económico una de las inequidades que persisten, en el seguro privado de salud, sea la diferencia en la cotización que deben pagar por su plan los hombres y mujeres (en edad fértil), pero nos parece que deben existir otras formas de abordar este problema en forma puntual, sin perder de vista una inequidad mayor aun, que existe para los adultos mayores, los cuales al terminar su vida activa no son capaces en su gran mayoría de mantener la cotización de los seguros privados, por el alto costo de ellos y deben migrar al sistema de seguro estatal (FONASA), sin importar el tiempo de permanencia en el seguro, así como tampoco la relación cotización - gasto real.

Nuestra sociedad en la que hoy convivimos, presenta un retraso evidente en lo ético y en lo legislativo, en relación al crecimiento tecnológico. De ahí la necesidad de reflexión, la necesidad de la búsqueda del bien de la sociedad, de aprender no solo lo que es posible de hacer, sino lo que es mejor hacer, para un individuo a veces, pero también para la sociedad en general. La búsqueda de la verdad, de lo que es correcto y verdadero, es una exigencia para Sociedades Científicas como la nuestra y para nuestra profesión.

Nuestra especialidad vive hoy cambios importantes que incluyen no solo los cambios demográficos que la afectan, la pérdida del encanto (como desafío al perfeccionamiento continuo y reconocimiento a la dedicación) al que tradicionalmente se asoció, sino también el número creciente de mujeres en los programas de formación de postgrado, y la necesidad de que nuestros especialistas se hagan cargo formalmente de la salud de la mujer. La protección de la salud de la mujer debemos ejercerla cuidando de los aspectos que en el pasado hemos entregado a otros especialistas: urólogos, coloproctólogos, oncólogos, radiólogos intervencionistas, médicos familiares. Los desafíos, entonces, que nuestra especialidad debe enfrentar suponen la reflexión necesaria para reformularla y definir lo que es mejor para nuestras pacientes, reforzando el concepto de los gíneco-obstetras como los profesionales al cuidado de la salud integral para la mujer.

Definitivamente otros son los desafíos de nuestra especialidad en el Chile de hoy. Ciertamente no es necesario incluir la atención del parto en la reforma de salud, porque nuestros indicadores son excelentes; porque la humanización no se establece por decreto; porque la tasa de cesárea y la humanización debemos abordarla desde la docencia a las futuras generaciones de gíneco-obstetras y matronas, sin descuidar la capacitación continua de todos los profesionales incluidos en la atención materna, en todo el país. Porque existen consecuencias en la práctica tradicional obstétrica, que de realizarse no sólo perjudicarían a nuestros especialistas, sino también a los establecimientos universitarios que hoy forman los profesionales que servirán a la población del país, y porque tales consecuencias favorecerían, aun más, cambios difícilmente reversibles en la relación médico-paciente.

Dr. ENRIQUe OYARZún E. Jefe Departamento de Obstetricia y Ginecología. Facultad de Medicina. Pontificia Universidad Católica de Chile.

Dr. EUGENIO SuÁREZ P. Vicepresidente, Sociedad Chilena de Obstetricia y Ginecología. Departamento de Obstetricia y Ginecología. Hospital San Borja-Arriarán, Universidad de Chile. 